\title{
A hydrodynamical model for covalent semiconductors with a generalized energy dispersion relation
}

\author{
GIUSEPPE ALİ ${ }^{1}$, GIOVANNI MASCALI ${ }^{1}$, \\ VITTORIO ROMANO ${ }^{2}$ and ROSA CLAUDIA TORCASIO ${ }^{1}$ \\ ${ }^{1}$ Dipartimento di Matematica, Università della Calabria \\ and INFN-Gruppo c. Cosenza, 87036 Cosenza, Italy \\ emails: giuseppe.ali@unical.it; g.mascali@unical.it; claudia.torcasio@yahoo.it \\ ${ }^{2}$ Dipartimento di Matematica e Informatica, Università di Catania, \\ viale A. Doria 6, 95125 Catania, Italy \\ email : romano@dmi.unict.it
}

(Received 22 February 2013; revised 28 December 2013; accepted 2 January 2014;

first published online 31 January 2014)

\begin{abstract}
We present the first macroscopical model for charge transport in compound semiconductors to make use of analytic ellipsoidal approximations for the energy dispersion relationships in the neighbours of the lowest minima of the conduction bands. The model considers the main scattering mechanisms charges undergo in polar semiconductors, that is the acoustic, polar optical, intervalley non-polar optical phonon interactions and the ionized impurity scattering. Simulations are shown for the cases of bulk $4 \mathrm{H}$ and $6 \mathrm{H}-\mathrm{SiC}$.
\end{abstract}

Key words: Compound semiconductors; Hydrodynamical models; Maximum entropy principle

\section{Introduction}

Lately, compound semiconductors have found wide use in the microelectronic industry. In particular, wider band gap semiconductors, such as $\mathrm{GaN}$ and $\mathrm{SiC}$, have attracted great interest, since they have a high breakdown field, a low thermal generation rate and a good thermal conductivity and stability. These properties are useful for high power and high temperature devices. For the description of the behaviour of these semiconductors there are Monte Carlo models [5, 15, 20,25], but there is a certain lack of macroscopic models, which are computationally less expensive [19]. Constructing a macroscopic model which is able to describe the behaviour of this type of semiconductor materials was the main aim of Ali et al. [1], and it is also the aim of the present paper, which can be considered as the natural continuation of the previous one. In particular, this required to address two crucial problems, that is, to model the band structure, and the scattering processes as much as possible. In Ali et al. [1] a spherical analytical approximation was used for energy dispersion relationships in the neighbours of the lowest minima of conduction bands (c.b.). In this paper we make use of an ellipsoidal approximation which is very important for describing carrier transport in semiconductors for which electron masses 
along the principal axes are consistently different, implying different carrier drift velocities along different directions. The plan of the paper is as follows. In Sections 2 and 3 we will briefly review the analytical approximation of the band structure of semiconductors and the kinetic semiclassical description of carrier transport. In Section 4 we will show how hydrodynamical models can be derived from the Boltzmann transport equations, and in Sections 5 and 6 we will find closure relations for these models by means of the maximum entropy principle (MEP). Eventually, in Section 7 numerical results for the cases of $4 \mathrm{H}-\mathrm{SiC}$ and $6 \mathrm{H}-\mathrm{SiC}$, which have anisotropic mass tensors, are shown and commented. The results are in good agreement with those in the literature, which are based on kinetic models.

\section{Analytic approximation of the band structure}

The electric charges which cause conductivity in semiconductors are the electrons which occupy the states around the minima of the lowest conduction bands, and analogously the holes in the states around the maxima of the highest valence bands. The dependence of the charge energy on the wave vector in the neighbours of these minima or maxima, which are called valleys, can be analytically approximated by non-parabolic dispersion relations of the following form [6]:

$$
\mathscr{E}_{A}\left(\mathbf{k}_{A}\right)=\frac{\hbar^{2}\left|\mathbf{k}_{A}\right|^{2}}{2 m_{e}} \gamma_{A}\left(\mathscr{E}_{A}\right) \psi_{A}^{-1}\left(\mathbf{n}_{A}\right),
$$

where $\mathscr{E}_{A}$ is the charge energy in the $A$-th valley measured from the bottom of the valley, the index $A$ running over the considered valleys, $\mathbf{k}_{A}$ is the electron quasi-wave vector referred for each valley to the minimum or maximum of the valley, $\mathbf{n}_{A}:=\frac{\mathbf{k}_{A}}{\left|\mathbf{k}_{A}\right|}, \gamma_{A}$ is the non-parabolicity factor, $\hbar$ is the reduced Planck constant and $m_{e}$ is the free electron mass. If one uses ellipsoidal approximation for electrons, the dependence of $\psi_{A}$ on $\mathbf{n}_{A}$ is given by

$$
\psi_{A}^{-1}=\frac{\left(\left(\mathbf{n}_{A}\right)_{1}\right)^{2}}{\left(m_{A}^{*}\right)_{1}}+\frac{\left(\left(\mathbf{n}_{A}\right)_{2}\right)^{2}}{\left(m_{A}^{*}\right)_{2}}+\frac{\left(\left(\mathbf{n}_{A}\right)_{3}\right)^{2}}{\left(m_{A}^{*}\right)_{3}},
$$

where $\left(m_{A}^{*}\right)_{i}^{-1}, i=1,2,3$, are the diagonal elements (eigenvalues) of the inverse effective mass tensor of the $A$-th valley, multiplied by $m_{e}$, referred to an orthonormal basis of the tensor.

Analogously for holes, one has

$$
\psi_{A}^{-1}=\left|A_{A}\right| \mp \sqrt{B_{A}^{2}+\frac{C_{A}^{2}}{4}\left(\sin ^{4} \vartheta \sin ^{2} 2 \varphi+\sin ^{2} 2 \vartheta\right)},
$$

in the case of warped bands, with $\varphi$ and $\theta$ respectively being azimuthal and polar angles, and $A_{A}, B_{A}$ and $C_{A}$ are the inverse valence band parameters.

Each valley in analytic approximations is extended to all $\mathbb{R}^{3}$, and the volume element in the k-space can be written as ${ }^{1}$

$$
d^{3} \mathbf{k}=\frac{m_{e} \sqrt{2 m_{e}}}{\hbar^{3}} \sqrt{\frac{\mathscr{E}}{\gamma^{5}(\mathscr{E})}}(\gamma(\mathscr{E})-\mathscr{E} \dot{\gamma}(\mathscr{E})) \psi^{\frac{3}{2}}(\varphi, \theta) d \mathscr{E} d \Omega,
$$

1 Henceforth we omit the valley index unless there is a possibility of confusion. 
where the dot denotes derivative with respect to the argument of the function, and $d \Omega$ is the solid angle element. The charge velocity, given by $\mathbf{v}=\frac{1}{\hbar} \nabla_{\mathbf{k}} \mathscr{E}$, has the following expression in terms of energy and angular variables,

$$
\begin{aligned}
v_{i} & =\frac{\hbar}{2 m_{e}} \frac{\gamma^{2}(\mathscr{E})}{\gamma(\mathscr{E})-\mathscr{E} \dot{\gamma}(\mathscr{E})} l_{i}, \quad l_{i}:=\frac{\partial}{\partial k_{i}}\left(|\mathbf{k}|^{2} \psi^{-1}\right)=g(\mathscr{E}) \eta_{i}(\varphi, \theta) n_{i}, \\
g: & =\frac{2 \sqrt{2 m_{e} \mathscr{E}}}{\hbar \sqrt{\gamma(\mathscr{E})}}, \quad \eta_{i}:=\frac{\sqrt{\psi}}{m_{i}^{*}} .
\end{aligned}
$$

In the following, for the sake of simplicity, we will consider only the case in which conductivity is essentially due to electrons, which happens for unipolar devices of n-type. Moreover, we will use the Kane approximation, according to which the non-parabolicity factor is

$$
\gamma=\frac{1}{1+\alpha \mathscr{E}}
$$

where the constant $\alpha$ is the non-parabolicity parameter.

\section{Semi-classical Boltzmann transport equations}

The electrons involved in electric conduction are semi-classically described as different populations according to the valley they belong to. Therefore, from a kinetic point of view, the electron state can be represented by a collection of distribution functions $f_{A}(\mathbf{x}, \mathbf{k}, t)$, whose time evolution is determined by a set of Boltzmann equations coupled among them and with the Poisson equation for the self-consistent electric potential $\phi[6]$,

$$
\begin{aligned}
& \frac{\partial f_{A}}{\partial t}+\mathbf{v}_{A} \cdot \nabla_{\mathbf{x}} f_{A}-\frac{q}{\hbar} \mathbf{E} \cdot \nabla_{\mathbf{k}} f_{A}=C_{A}\left[f_{A}\right]+\sum_{B \neq A} C_{A B}\left[f_{A}, f_{B}\right], \\
& -\nabla_{\mathbf{x}} \cdot\left(\epsilon_{S} \nabla_{\mathbf{x}} \phi\right)=q\left[N_{D}(\mathbf{x})-n(\mathbf{x})\right],
\end{aligned}
$$

where $\mathrm{q}$ is the absolute value of the electron charge, $\mathbf{E}=-\nabla_{\mathbf{x}} \phi$ is the electric field, $\epsilon_{S}$ is the dielectric constant of a semiconductor, $N_{D}(\mathbf{x})$ is the doping concentration, $n_{V}$ is the number of valleys and $n=\sum_{A} \int_{\mathbb{R}^{3}} f_{A}(\mathbf{x}, \mathbf{k}, t) d^{3} \mathbf{k}$ is the total electron density. The right-hand side of equation (3.1) is due to the collisions that electrons undergo in a semiconductor. These can substantially be divided into the following two classes, corresponding to the terms $C_{A}$ and $C_{A B}$ respectively: those which leave electrons in the same valley as they were before the collision (intra-valley transitions), and those which drive electrons into a different valley (inter-valley transitions).

Neglecting degeneration, collision operators belonging to the first class can be written in the form

$$
C_{A}[f]=\int_{\mathbb{R}^{3}}\left[P_{A}\left(\mathbf{k}^{\prime}, \mathbf{k}\right) f\left(\mathbf{k}^{\prime}\right)-P_{A}\left(\mathbf{k}, \mathbf{k}^{\prime}\right) f(\mathbf{k})\right] d \mathbf{k}^{\prime},
$$

where $P_{A}\left(\mathbf{k}^{\prime}, \mathbf{k}\right)$ is the transition rate from the state with quasi-wave vector $\mathbf{k}^{\prime}$ to the state with quasi-wave vector $\mathbf{k}$, both belonging to the same $A$-th valley. 
The collision operators of the second class have the form

$$
C_{A B}\left[f_{A}, f_{B}\right]=\int_{\mathbb{R}^{3}}\left[P_{B A}\left(\mathbf{k}_{B}^{\prime}, \mathbf{k}_{A}\right) f_{B}^{\prime}-P_{A B}\left(\mathbf{k}_{A}, \mathbf{k}_{B}^{\prime}\right) f_{A}\right] d^{3} \mathbf{k}_{B}^{\prime},
$$

where $P_{A B}\left(\mathbf{k}_{A}, \mathbf{k}_{B}^{\prime}\right)$ is the transition rate from the state with quasi-wave vector $\mathbf{k}_{A}$ belonging to the $A$-th valley to the state with quasi-wave vector $\mathbf{k}_{B}^{\prime}$ belonging to the $B$-th valley. The only difference with the previous class is that now $A$ and $B$ refer to different valleys.

The form of the scattering rates $P_{A}\left(\mathbf{k}, \mathbf{k}^{\prime}\right)$ and $P_{B A}\left(\mathbf{k}_{B}, \mathbf{k}_{A}^{\prime}\right)$ depends on the mechanism of interaction. Here we will consider polar optical and acoustic phonon scattering, and impurity scattering regarding the first class of operators, and non-polar optical phonon scattering for the second class. The scattering rates for the above-mentioned scattering mechanisms are summarized below, see Jacoboni \& Lugli [6].

The acoustic phonon scattering transition rate in its elastic approximation reads

$$
P^{(\mathrm{ac})}\left(\mathbf{k}, \mathbf{k}^{\prime}\right)=\mathscr{K}^{(\mathrm{ac})} \delta\left(\mathscr{E}\left(\mathbf{k}^{\prime}\right)-\mathscr{E}(\mathbf{k})\right)
$$

with $\mathscr{K}^{(\mathrm{ac})}=\frac{k_{B} T_{L} \Xi_{d}^{2}}{4 \pi^{2} \hbar \rho v_{s}^{2}}$. Here $k_{B}$ is the Boltzmann constant, $T_{L}$ is the lattice temperature, taken to be constant, $\Xi_{d}$ is the acoustic deformation potential, $\rho$ is the material density and $v_{s}$ is the sound velocity.

The polar optical phonon scattering transition rate is ${ }^{2}$

$$
P^{(p)}\left(\mathbf{k}, \mathbf{k}^{\prime}\right)=\frac{\mathscr{K}^{(p)}}{\left|\mathbf{k}-\mathbf{k}^{\prime}\right|^{2}} \mathscr{G}\left(\mathbf{k}, \mathbf{k}^{\prime}\right)\left[\begin{array}{c}
N^{(p)} \\
N^{(p)}+1
\end{array}\right] \delta\left(\mathscr{E}^{\prime}-\mathscr{E} \mp \hbar \omega^{(p)}\right),
$$

with $\mathscr{K}^{(p)}=\frac{q^{2} \omega^{(p)}}{8 \pi^{2}}\left(\frac{1}{\epsilon_{\infty}}-\frac{1}{\epsilon_{s}}\right)$. Here $\omega^{(p)}$ is the polar optical phonon frequency, $N^{(p)}$ is the Bose-Einstein polar optical phonon occupation number, $\epsilon_{\infty}$ is the high frequency dielectric constant and $\mathscr{G}\left(\mathbf{k}, \mathbf{k}^{\prime}\right)$ is the overlap factor.

The impurity scattering transition rate reads as

$$
P^{(\mathrm{im})}\left(\mathbf{k}, \mathbf{k}^{\prime}\right)=\mathscr{K}^{(\mathrm{im})} \frac{\mathscr{G}\left(\mathbf{k}, \mathbf{k}^{\prime}\right)}{\left[\left|\mathbf{k}-\mathbf{k}^{\prime}\right|^{2}+\lambda_{D}^{2}\right]^{2}} \delta\left(\mathscr{E}^{\prime}-\mathscr{E}\right)
$$

with $\lambda_{D}=\sqrt{\frac{N_{D} q^{2}}{\epsilon_{s} k_{B} T_{L}}}$, the inverse Debye length, and $\mathscr{K}^{(\mathrm{im})}=\frac{Z^{2} N_{D} q^{4}}{4 \pi \hbar \epsilon_{s}^{2}}$, where $Z$ is the impurity charge number.

Eventually, the non-polar optical inter-valley scattering rate is given by

$$
P_{A B}^{(\mathrm{np})}\left(\mathbf{k}_{A}, \mathbf{k}_{B}^{\prime}\right)=\mathscr{K}^{(\mathrm{np})} Z_{A B}\left[\begin{array}{c}
N^{(\mathrm{np})} \\
N^{(\mathrm{np})}+1
\end{array}\right] \delta\left(\mathscr{E}_{B}^{\prime}-\mathscr{E}_{A}-\Delta_{A B} \mp \hbar \omega^{(\mathrm{np})}\right),
$$

with $A \neq B$, and $\mathscr{K}^{(\mathrm{np})}=\frac{\left(D_{t} K\right)^{2}}{8 \pi^{2} \rho \omega^{(\mathrm{np})}}$. Here $D_{t} K$ is the inter-valley deformation potential, $Z_{A B}$ is the number of equivalent final valleys $B$ as seen from the initial valley $A[23], \omega^{(\mathrm{np})}$ is

2 The short notation $\left[\begin{array}{c}N^{(p)} \\ N^{(p)}+1\end{array}\right] \delta\left(\mathscr{E}^{\prime}-\mathscr{E} \mp \hbar \omega^{(p)}\right)$ stands for $N^{(p)} \delta\left(\mathscr{E}^{\prime}-\mathscr{E}-\hbar \omega^{(p)}\right)+\left(N^{(p)}+1\right) \delta\left(\mathscr{E}^{\prime}-\right.$ $\left.\mathscr{E}+\hbar \omega^{(p)}\right)$. 
the inter-valley phonon frequency, $\Delta_{A B}=\mathscr{E}_{A}^{(0)}-\mathscr{E}_{B}^{(0)}$, with $\mathscr{E}_{A}^{(0)}$ being the energy minimum of the $A$-th valley and $N^{(\mathrm{np})}$ has an obvious meaning.

\section{Hydrodynamical model}

The semiclassical kinetic model correctly describes the charge transport in semiconductors up to when quantum effects are negligible. However, this model is computationally very expensive, therefore the introduction of macroscopic models can be very useful for computer-aided design (CAD) purposes. These models can be obtained by introducing the moments of the distribution functions, which for a weight function $\alpha(\mathbf{k})$ are defined as

$$
M_{\alpha}(\mathbf{x}, t):=\int_{\mathbb{R}^{3}} \alpha(\mathbf{k}) f(\mathbf{x}, \mathbf{k}, t) d^{3} \mathbf{k}
$$

As in the previous paper [1], we will consider the weight functions $1, \mathbf{v}(\mathbf{k}), \mathscr{E}(\mathbf{k})$ and $\mathscr{E}(\mathbf{k}) \mathbf{v}(\mathbf{k})$, to which the following macroscopic quantities correspond, all having direct physical meaning,

$$
\begin{aligned}
n & =M_{1}, & & \text { electron density, } \\
\mathbf{V} & =\frac{1}{n} M_{\mathbf{v}}, & & \text { electron mean velocity, } \\
W & =\frac{1}{n} M_{\mathscr{E}}, & & \text { electron mean energy, } \\
\mathbf{S} & =\frac{1}{n} M_{\mathscr{E} \mathbf{v}}, & & \text { electron mean energy flux. }
\end{aligned}
$$

Of course, there is a set of such quantities for each electron population. The evolution equations of these state variables are obtained by taking the moments of the Boltzmann equations. So doing, one has

$$
\begin{gathered}
\frac{\partial n_{A}}{\partial t}+\sum_{j} \frac{\partial\left(n_{A} V_{A}^{j}\right)}{\partial x^{j}}=C_{n_{A}}, \\
\frac{\partial\left(n_{A} V_{A}^{i}\right)}{\partial t}+\sum_{j} \frac{\partial\left(n_{A} F_{A}^{(0) i j}\right)}{\partial x^{j}}+q n_{A} \sum_{j} E_{j} G_{A}^{(0) i j}=n_{A} C_{V_{A}}^{i}, \quad i=1,2,3, \\
\frac{\partial\left(n_{A} W_{A}\right)}{\partial t}+\sum_{j} \frac{\partial\left(n_{A} S_{A}^{j}\right)}{\partial x^{j}}+q n_{A} \sum_{j} E_{j} V_{A}^{j}=C_{W_{A}}, \\
\frac{\partial\left(n_{A} S_{A}^{i}\right)}{\partial t}+\sum_{j} \frac{\partial\left(n_{A} F_{A}^{(1) i j}\right)}{\partial x^{j}}+q n_{A} \sum_{j} E_{j} G_{A}^{(1) i j}=n_{A} C_{S_{A}}^{i}, \quad i=1,2,3,
\end{gathered}
$$


where $A$ runs over the considered valleys. In the above equations

$$
\begin{aligned}
& C_{n_{A}}=\int\left(C\left[f_{A}\right]+\sum_{B \neq A} C_{A B}\left[f_{A}, f_{B}\right]\right) d^{3} \mathbf{k} \quad \text { is the density production, } \\
& F_{A}^{(0) i j}=\frac{1}{n_{A}} \int v_{A}^{i} v_{A}^{j} f_{A} d^{3} \mathbf{k} \quad \text { is the velocity flux, } \\
& G_{A}^{(0) i j}=\frac{1}{n_{A}} \int \frac{1}{\hbar} \frac{\partial v_{A}^{i}}{\partial k^{j}} f_{A} d^{3} \mathbf{k}, \\
& C_{V_{A}}^{i}=\frac{1}{n_{A}} \int v_{A}^{i}\left(C\left[f_{A}\right]+\sum_{B \neq A} C_{A B}\left[f_{A}, f_{B}\right]\right) d^{3} \mathbf{k} \text { is the velocity production, } \\
& C_{W_{A}}=\int \mathscr{E}_{A}(\mathbf{k})\left(C\left[f_{A}\right]+\sum_{B \neq A} C_{A B}\left[f_{A}, f_{B}\right]\right) d^{3} \mathbf{k} \text { is the energy production, } \\
& F_{A}^{(1) i j}=\frac{1}{n_{A}} \int \mathscr{E}_{A}(\mathbf{k}) v_{A}^{i} v_{A}^{j} f_{A} d^{3} \mathbf{k} \quad \text { is the flux of the energy flux, } \\
& G_{A}^{(1) i j}=\frac{1}{n_{A}} \int \frac{1}{\hbar} \frac{\partial\left(\mathscr{E}_{A} v_{A}^{i}\right)}{\partial k^{j}} f_{A} d^{3} \mathbf{k}, \\
& C_{S_{A}}^{i}=\frac{1}{n_{A}} \int \mathscr{E} A v_{A}^{i}\left(C\left[f_{A}\right]+\sum_{B \neq A} C_{A B}\left[f_{A}, f_{B}\right]\right) d^{3} \mathbf{k} \text { is the energy flux production. }
\end{aligned}
$$

The system of equations (4.2)-(4.5) is not closed, since the fluxes $F_{A}^{(0) i j}, G_{A}^{(0) i j}, F_{A}^{(1) i j}$ and $G_{A}^{(1) i j}$ appear as do the production terms $C_{n_{A}}, C_{V_{A}}^{i}, C_{W_{A}}$ and $C_{S_{A}}^{i}$, which are extra variables to be expressed in terms of the state variables $n_{A}, \mathbf{V}_{A}, W_{A}$ and $\mathbf{S}_{A}$.

In the next two sections we will show how the system can be closed by using the maximum entropy distribution functions, linearized with respect to the vector variables. So doing, the fluxes will be expressed by diagonal matrices whose elements depend only on the macroscopic energies (6.2)-(6.4). Also, the production terms for the densities and the energies will depend only on the energies (6.8) and (6.12), while those for the velocities and energy fluxes will be linear combinations of $\mathbf{V}$ and $\mathbf{S}$, with coefficients depending on energies, see (6.5), (6.6), (6.9), (6.10), (6.11) and (6.13) for the matrices of these coefficients. We emphasise that, for each valley, the non-scalar Lagrange multipliers and the extra variables will be expressed in terms of their components in the basis consisting of the unit vectors parallel to the principal crystallographic directions of the valley.

\section{Closure of the moment equations by the maximum entropy principle}

In order to get the closure relations, we will make use of MEP, which has been successfully applied in many fields of physics, and particularly in semiconductor modelling (see [2, $3,7,10-12,17,18$ ], and references therein). According to this principle, if a certain number of moments are known, then the distribution functions $f_{A}^{\mathrm{MEP}}$, which can be used for evaluating the unknown moments, are those that minimise or maximise the entropy functional under the constraint that those functions reproduce the known moments. In our case this is justified by the fact that, since the phonon gas is considered as a thermal bath at constant temperature $T_{L}$, the Helmholtz free energy is a Lyapunov functional [9], and it can be shown immediately that the consequent optimization problem is equivalent 
to finding stationary points of only the electron component of the entropy, with the average energy being one of the constraints. Moreover, if the electron gas is sufficiently dilute, one can take the expression of the entropy obtained as a limiting case of that arising from the Fermi statistics,

$$
\mathscr{S}=-k_{B} \sum_{A} \int_{\mathbb{R}^{3}}\left(f_{A} \log \frac{f_{A}}{y}-f_{A}\right) d^{3} \mathbf{k}, \quad y=\frac{2}{(2 \pi)^{3}} .
$$

Therefore, according to MEP, we can estimate $f_{A}$ 's with the distributions $f_{A}^{\mathrm{MEP}}$ 's that solve the problem,

$$
\text { optimise } \mathscr{S} \text { under the constraints } M_{\alpha}^{A}=\int_{\mathbb{R}^{3}} \alpha(\mathbf{k}) f_{A}^{\mathrm{MEP}} d^{3} \mathbf{k}, \quad \alpha=1, \mathbf{v}, \mathscr{E}, \mathscr{E} \mathbf{v} \text {. }
$$

The resulting maximum entropy distribution functions read (the factors $k_{B}$ and $y$ have been included into the multipliers) as

$$
f_{A}^{\mathrm{MEP}}=\exp \left[-\left(\lambda^{A}+\lambda_{\mathrm{V}}^{A} \cdot \mathbf{v}_{A}+\left(\lambda_{W}^{A}+\lambda_{\mathrm{S}}^{A} \cdot \mathbf{v}_{A}\right) \mathscr{E}_{A}\right)\right], \quad A=1,2 \ldots, n_{V}
$$

These have to be inserted into the constraint relations and, in principle, after inversion, the Lagrangian multipliers can be expressed as functions of the basic moments $n_{A}, \mathbf{V}_{A}$, $W_{A}$ and $\mathbf{S}_{A}$. However, an analytic inversion is impossible and a numerical one is not practical for numerical simulations of electron devices, since it has to be performed at each time step in the evolution of state variables (see [8] for Si semiconductors). Using the same approach as in $[13,14,22]$, we assume a small anisotropy of distribution functions and linearize them with respect to the vector multipliers,

$$
f_{A}^{\mathrm{MEP}} \approx \exp \left[-\lambda^{A}-\lambda_{W}^{A} \mathscr{E}_{A}\right]\left[1-\left(\lambda_{\mathrm{V}}^{A} \cdot \mathbf{v}_{A}+\lambda_{\mathrm{S}}^{A} \cdot \mathbf{v}_{A} \mathscr{E}_{A}\right)\right], \quad A=1,2 \ldots, n_{V}
$$

Then the constraints reduce to

$$
\begin{aligned}
n_{A} & =\int_{\mathbb{R}^{3}} f_{A}^{(0)} d^{3} \mathbf{k}, \quad n_{A} \mathbf{v}_{A}=-\int_{\mathbb{R}^{3}} \mathbf{v}_{A}\left(\lambda_{\mathbf{V}}^{A} \cdot \mathbf{v}_{A}+\lambda_{\mathbf{S}}^{A} \cdot \mathbf{v}_{A} \mathscr{E}_{A}\right) f_{A}^{(0)} d^{3} \mathbf{k}, \\
n_{A} W_{A} & =\int_{\mathbb{R}^{3}} \mathscr{E}_{A} f_{A}^{(0)} d^{3} \mathbf{k}, \quad n_{A} \mathbf{S}_{A}=-\int_{\mathbb{R}^{3}} \mathscr{E}_{A} \mathbf{v}_{A}\left(\lambda_{\mathbf{V}}^{A} \cdot \mathbf{v}_{A}+\lambda_{\mathbf{S}}^{A} \cdot \mathbf{v}_{A} \mathscr{E}_{A}\right) f_{A}^{(0)} d^{3} \mathbf{k},
\end{aligned}
$$

with $f_{A}^{(0)}=\exp \left[-\lambda^{A}-\lambda_{W}^{A} \mathscr{E}_{A}\right]$. In this way, the scalar constraints can be solved for the scalar Lagrange multipliers as functions of scalar moments. The vector constraints reduce to linear relationships between the vector Lagrange multipliers and the vector moments. In the next section functions (5.1) will be used to find the closure relations.

\section{Closure relations for the fluxes and the production terms}

We will start this section by inverting the constraint relations obtained with approximate maximum entropy distribution functions. Since, in general, the angular integration cannot be performed analytically when the ellipsoidal approximations are used for the energy dispersion relations, computations are still more involved than those in [1]. From the 
scalar constraints, the densities and the energies are given by

$$
\begin{array}{r}
n=\frac{m_{e} \sqrt{2 m_{e}} J_{0}}{\hbar^{3}} e^{-\lambda} d_{0}\left(\lambda_{W}\right), \\
n W=\frac{m_{e} \sqrt{2 m_{e}} J_{0}}{\hbar^{3}} e^{-\lambda} d_{1}\left(\lambda_{W}\right),
\end{array}
$$

where $d_{n}\left(\lambda_{W}\right):=\int_{0}^{\infty} \mathscr{E} n \exp \left(-\lambda_{W} \mathscr{E}\right) \sqrt{\frac{\mathscr{E}}{\gamma^{5}(\mathscr{E})}}(\gamma(\mathscr{E})-\mathscr{E} \dot{\gamma}(\mathscr{E})) d \mathscr{E}$, and $J_{0}:=\int_{S^{2}} \psi^{\frac{3}{2}} d \Omega$. Thus, one has

$$
\lambda=-\log \left(\frac{\hbar^{3} n}{m_{e} \sqrt{2 m_{e}} J_{0} d_{0}}\right), \quad \lambda_{W}=h^{-1}(W)
$$

$S^{2}$ being the unit sphere surface, and $h^{-1}$ is the inverse function of $h\left(\lambda_{W}\right):=\frac{d_{1}\left(\lambda_{W}\right)}{d_{0}\left(\lambda_{W}\right)}$. Hereafter $\lambda_{W}$ will always be regarded as a function of $W$.

As regards the vector Lagrange multipliers, one finds

$$
\lambda_{V_{i}}=J_{1, i}^{-1}\left[b_{11}(W) V_{i}+b_{12}(W) S_{i}\right], \quad \lambda_{S_{i}}=J_{1, i}^{-1}\left[b_{12}(W) V_{i}+b_{22}(W) S_{i}\right] .
$$

Here $J_{1, i}:=\int_{S^{2}} \psi^{\frac{3}{2}} \eta_{i}^{2} n_{i}^{2} d \Omega$ and $b_{i j}$ are the elements of matrix $\mathbf{B}$, which is the inverse of the symmetric matrix $\mathbf{A}$ of elements

$a_{i j}=-\frac{\hbar^{2}}{4 m_{e}^{2} J_{0}} \frac{p_{i+j-2}}{d_{0}}, i, j=1,2$, with $p_{n}=p_{n}(W):=\int_{\mathbb{R}^{3}} \frac{\mathscr{E}^{n+\frac{1}{2}}}{\gamma(\mathscr{E})-\mathscr{E} \dot{\gamma}(\mathscr{E})} \gamma^{\frac{3}{2}}(\mathscr{E}) g^{2}(\mathscr{E}) e^{-\lambda_{W} \mathscr{E}} d \mathscr{E}$

Matrix $\mathbf{B}$ is symmetric, which reminds us of the Onsager relations [16].

We note that, differently from the findings in the isotropic approximation [1], relations (6.1) depend on the direction due to the presence of the terms $J_{1, i}^{-1}$, which in the anisotropic case are different for $i=1,2,3$.

For the results in this and in the following sections, we have used the following.

Property 1 If $\sigma(\mathbf{n})$ is an integrable even function of its argument then

$$
\begin{gathered}
\int_{S^{2}} \sigma(\mathbf{n}) n_{i} d \Omega=0, \quad \int_{S^{2}} \sigma(\mathbf{n}) n_{i} n_{j} d \Omega=0, \quad i, j=1,2,3, i \neq j . \\
\int_{S^{2}} \sigma(\mathbf{n}) n_{i} n_{j} n_{k} d \Omega=0, \quad \forall i, j, k=1,2,3 .
\end{gathered}
$$

\subsection{Fluxes}

The above-written relations for the Lagrange multipliers allow us to find constitutive equations for fluxes. We obtain

$$
\left(\begin{array}{c}
F_{i j}^{(0)} \\
F_{i j}^{(1)}
\end{array}\right)=\frac{\hbar^{2} J_{1, i}}{4 m_{e}^{2} J_{0} d_{0}}\left(\begin{array}{c}
p_{0} \\
p_{1}
\end{array}\right) \delta_{i j}
$$


with $\delta_{i j}$ being the Kronecker delta, and

$$
\begin{aligned}
G_{i j}^{(0)} & =\frac{\hbar}{\left(2 m_{e}\right)^{\frac{3}{2}} J_{0} d_{0}}\left[\frac{\hbar J_{1, i}}{\sqrt{2 m_{e}}} \mathscr{G}_{1,0}(W)+\left(J_{3, i}-J_{2, i}\right) \mathscr{G}_{2,0}(W)\right] \delta_{i j} \\
G_{i j}^{(1)} & =\frac{\hbar}{\left(2 m_{e}\right)^{\frac{3}{2}} J_{0} d_{0}}\left[\frac{\hbar J_{1, i}}{\sqrt{2 m_{e}}} \mathscr{G}_{1,1}(W)+\left(J_{3, i}-J_{2, i}\right) \mathscr{G}_{2,1}(W)+\frac{\hbar J_{1, i}}{\sqrt{2 m_{e}}} \mathscr{G}_{3,0}(W)\right] \delta_{i j},(6.4)
\end{aligned}
$$

where

$$
\begin{aligned}
\mathscr{G}_{1, n}(W): & \int_{0}^{\infty} \mathscr{E} \sqrt{\frac{\mathscr{E}}{\gamma(\mathscr{E})}} g(\mathscr{E}) \dot{\beta}(\mathscr{E}) \exp \left(-\lambda_{W} \mathscr{E}\right) d \mathscr{E}, \\
\mathscr{G}_{2, n}(W): & \int_{0}^{\infty} \mathscr{E}^{\mathscr{n}} g(\mathscr{E}) \exp \left(-\lambda_{W} \mathscr{E}\right) d \mathscr{E}, \\
\mathscr{G}_{3, n}(W): & \int_{0}^{\infty} \mathscr{E}^{\mathscr{E}} \sqrt{\frac{\mathscr{E}}{\gamma(\mathscr{E})}} g(\mathscr{E}) \beta(\mathscr{E}) \exp \left(-\lambda_{W} \mathscr{E}\right) d \mathscr{E}, \\
J_{2, i} & :=\int_{S^{2}}\left(\eta_{i}-2 \frac{\partial \eta_{i}}{\partial n_{i}}\right) n_{i}^{2} \psi d \Omega, \quad J_{3, i}:=\int_{S^{2}}\left(\eta_{i}-2 \sum_{j} \frac{\partial \eta_{i}}{\partial n_{j}^{2}} n_{j}^{2} n_{i}^{2}\right) \psi d \Omega
\end{aligned}
$$

with $\beta:=\frac{\gamma^{2}}{\gamma-\mathscr{\mathscr { \gamma }} \dot{\gamma}} g$.

\subsection{Production terms: acoustic phonon scattering}

Being intra-valley and elastic, the only non-zero production terms for the acoustic scattering (3.3) are those relative to the velocities and the energy fluxes. After some calculations we find

$$
\left(\begin{array}{l}
C_{\mathrm{V}}^{(\mathrm{ac})} \\
C_{\mathbf{S}}^{(\mathrm{ac})}
\end{array}\right)=\mathbf{C}^{(\mathrm{ac})}\left(\begin{array}{c}
\mathbf{V} \\
\mathbf{S}
\end{array}\right)
$$

where $\mathbf{C}^{(\mathrm{ac})}:=\mathbf{Q}^{(\mathrm{ac})} \mathbf{B}$ and $\mathbf{Q}^{(\mathrm{ac})}$ is the matrix of elements

$$
q_{i j}^{(\mathrm{ac})}(W)=\frac{1}{2 \sqrt{2 m_{e}} \hbar d_{0}} \mathscr{K}^{(\mathrm{ac})} \int_{0}^{\infty} \mathscr{E}^{i+j-1} \frac{g^{2}(\mathscr{E})}{\gamma(\mathscr{E})} \exp \left(-\lambda_{W} \mathscr{E}\right) d \mathscr{E} .
$$

We note that at the level of the approximation used in this paper, the contribution of acoustic scattering is isotropic.

\subsection{Production terms: polar optical phonon scattering}

For the polar optical scattering (3.4), the calculations are very involved, since it is not possible to separate angular integration from energy integration because of the presence of the overlap factor $\mathscr{G}$. It is necessary to make some suitable assumptions on this factor, which, through $\mathbf{k}$ and $\mathbf{k}^{\prime}$, depends on the carrier energies, $\mathscr{E}$ and $\mathscr{E}$, and the motion directions, $\mathbf{n}$ and $\mathbf{n}^{\prime}$, before and after the scattering. 
Table 1. Parameter values used in the overlap model

\begin{tabular}{cc}
\hline \hline Parameter & Value \\
\hline$a_{01}$ & 0.873 \\
$a_{02}$ & 0.0268 \\
$b_{01}$ & $4.989 \times 10^{-7} \mu \mathrm{m}^{2}$ \\
$b_{02}$ & $-4.250 \times 10^{-7} \mu \mathrm{m}^{2}$ \\
\hline \hline
\end{tabular}

Assumption 1 We will assume that $\mathscr{G}$ can depend on $\mathbf{n}$ and $\mathbf{n}^{\prime}$ only through the scalar product $\mathbf{n} \cdot \mathbf{n}^{\prime}$, even functions of $n_{i}$ and $n_{i}^{\prime}, i=1,2,3$, and also through $n_{z}-n_{z}^{\prime}$, as long as this latter dependence is even too.

This assumption is satisfied by both the expression of $\mathscr{G}$ used by Fawcett et al. [4] and the one proposed by Nilsson et al. [20], which we will use in this paper, that is

$$
\mathscr{G}\left(\mathbf{k}, \mathbf{k}^{\prime}\right)=1-\left(a_{01}+a_{02} \sin \chi\right)\left[1-\exp \left[-\left(b_{01}+b_{02} \sin \chi\right)|\mathbf{q}|^{2}\right],\right.
$$

where $\mathbf{q}=\mp\left(\mathbf{k}^{\prime}-\mathbf{k}\right)$ is the phonon quasi-wave vector and $\chi:=\arccos \frac{q_{z}}{|\mathbf{q}|}$, while the constants $a_{01}, a_{02}, b_{01}$ and $b_{02}$ can be found in Table 1 .

Under Assumption 1, the following properties hold:

Property 2 The function

$$
G^{s, p}\left(\mathscr{E}, \mathscr{E}^{\prime}, \mathbf{n}\right):=\int_{S^{2}} \frac{\mathscr{G}\left(\mathbf{k}, \mathbf{k}^{\prime}\right)}{\frac{\mathscr{E}}{\gamma(\mathscr{E})} \psi(\mathbf{n})+\frac{\mathscr{E}^{\prime}}{\gamma\left(\mathscr{E}^{\prime \prime}\right)} \psi\left(\mathbf{n}^{\prime}\right)-2 \sqrt{\frac{\mathscr{E} \mathscr{E}^{\prime}}{\gamma(\mathscr{E}) \gamma(\mathscr{E})} \psi(\mathbf{n}) \psi\left(\mathbf{n}^{\prime}\right)} \mathbf{n} \cdot \mathbf{n}^{\prime}} \psi^{\frac{3}{2}}\left(\mathbf{n}^{\prime}\right) d \Omega^{\prime}
$$

is even with respect to $n_{i}, i=1,2,3$.

Property 3 The functions

$$
\begin{aligned}
& G_{i}^{v, p}\left(\mathscr{E}, \mathscr{E}^{\prime}, \mathbf{n}\right) \\
& :=\int_{S^{2}} \frac{\mathscr{G}\left(\mathbf{k}, \mathbf{k}^{\prime}\right)}{\frac{\mathscr{E}}{\gamma(\mathscr{E})} \psi(\mathbf{n})+\frac{\mathscr{E}}{\gamma\left(\mathscr{E}^{\prime}\right)} \psi\left(\mathbf{n}^{\prime}\right)-2 \sqrt{\frac{\mathscr{\mathscr { E }} \mathscr{E}^{\prime}}{\gamma(\mathscr{E}) \gamma\left(\mathscr{E}^{\prime}\right)} \psi(\mathbf{n}) \psi\left(\mathbf{n}^{\prime}\right)} \mathbf{n} \cdot \mathbf{n}^{\prime}} \psi^{\frac{3}{2}}\left(\mathbf{n}^{\prime}\right) \eta_{i}\left(\mathbf{n}^{\prime}\right) n_{i}^{\prime} d \Omega^{\prime}, i=1,2,3
\end{aligned}
$$

are odd with respect to $n_{i}$ and even with respect to $n_{j}, j \neq i, i, j=1,2,3$.

Function $G^{s, p}\left(\mathscr{E}, \mathscr{E}^{\prime}, \mathbf{n}\right)$ takes into account the angular integration of the core term $\frac{\mathscr{G}\left(\mathbf{k}, \mathbf{k}^{\prime}\right)}{\left|\mathbf{k}-\mathbf{k}^{\prime}\right|^{2}}$, appearing in the expression of $P^{(p)}\left(\mathbf{k}, \mathbf{k}^{\prime}\right)$, given by (3.4). Function $G_{i}^{v, p}(\mathscr{E}, \mathscr{E}, \mathbf{n})$ shows up in the angular integration of the term $\frac{\mathscr{G}\left(\mathbf{k}, \mathbf{k}^{\prime}\right)}{\left|\mathbf{k}-\mathbf{k}^{\prime}\right|^{2}} v_{i}(\mathbf{k})$. Then the second angular integration which is needed for the evaluation of production terms can be simplified by using Property 1 . We also use the detailed balance principle, which here we write in the general form valid for inter-valley scattering too:

$$
P_{B A}\left(\mathbf{k}_{B}^{\prime}, \mathbf{k}_{A}\right)=P_{A B}\left(\mathbf{k}_{A}, \mathbf{k}_{B}^{\prime}\right) \exp \left(-\frac{\mathscr{E}_{A}-\mathscr{E}_{B}^{\prime}+\Delta_{A B}}{k_{B} T_{L}}\right)
$$


As regards the density and energy production terms, we find

$$
\begin{aligned}
C_{n}^{(p)}= & 0, \\
C_{W}^{(p)}= & \frac{\sqrt{m_{e}} \omega^{(p)}}{\sqrt{2} J_{0} d_{0}} N^{(p)} \mathscr{K}^{(p)} n \int_{0}^{\infty}\left(\int_{S^{2}} G^{s, p}\left(\mathscr{E}, \mathscr{E}^{+}, \mathbf{n}\right) \psi^{\frac{3}{2}}(\mathbf{n}) d \Omega\right) H_{1}(\mathscr{E}) H_{1}\left(\mathscr{E}^{+}\right) \\
& \times\left[1-e^{-\hbar \omega^{(p)}\left(\lambda_{W}-\frac{1}{k_{B} T_{L}}\right)}\right] \exp \left(-\lambda_{W} \mathscr{E}\right) d \mathscr{E},
\end{aligned}
$$

where $\mathscr{E}^{+}:=\mathscr{E}+\hbar \omega^{(p)}$, and function $H_{1}$ is as given in Appendix A.

The structure of production terms for velocities and energy fluxes is slightly different from that for acoustic scattering, that is, it is anisotropic

$$
\left(\begin{array}{c}
C_{V_{i}}^{(p)} \\
C_{S_{i}}^{(p)}
\end{array}\right)=\mathbf{C}^{(p), i}\left(\begin{array}{c}
V_{i} \\
S_{i}
\end{array}\right)
$$

where $\mathbf{C}^{(p), i}:=J_{1, i}^{-1} \mathbf{Q}^{(p)} \mathbf{B}, i=1,2,3$, and $\mathbf{Q}^{(p)}$ is the matrix of elements

$$
\begin{aligned}
& q_{i j}^{(p)}=\frac{\hbar}{4 m_{e} \sqrt{2 m_{e}} J_{0} d_{0}} N^{(p)} \mathscr{K}^{(p)} \int_{0}^{\infty}\left[H_{2, i}\left(\mathscr{E}, \mathscr{E}^{+}\right) \mathscr{E}^{\mathscr{i + j - 1}}+H_{3, i}\left(\mathscr{E}, \mathscr{E}^{+}\right)\left(\mathscr{E}^{+}\right)^{i+j-1}\right. \\
& \left.-H_{4, i}\left(\mathscr{E}, \mathscr{E}^{+}\right)\left(e^{\hbar \omega^{(p)}\left(\frac{1}{k_{B} T_{L}}-\lambda_{W}\right)} \mathscr{\mathscr { E }}^{i-1}\left(\mathscr{E}^{+}\right)^{j-1}+\left(\mathscr{E}^{+}\right)^{i-1} \mathscr{E}^{j-1}\right)\right] e^{-\lambda_{W} \mathscr{E}} d \mathscr{E},
\end{aligned}
$$

and functions $H_{2, i}, H_{3, i}$ and $H_{4, i}, i=1,2,3$, are also given in Appendix A.

\subsection{Production terms: impurity scattering}

For the impurity scattering (3.5), the procedure is very similar to that followed for polar optical scattering, in fact properties analogous to Properties 2 and 3, respectively, hold for the following functions:

$\begin{aligned} G^{s, i m}(x, \mathbf{n}) & :=\int_{S^{2}} \frac{\mathscr{G}\left(\mathbf{k}, \mathbf{k}^{\prime}\right) \psi^{\frac{3}{2}}\left(\mathbf{n}^{\prime}\right)}{\left[\frac{2 m_{e} \mathscr{E}}{\hbar^{2} \gamma(\mathscr{E})}\left(\psi(\mathbf{n})+\psi\left(\mathbf{n}^{\prime}\right)-2 \sqrt{\psi(\mathbf{n}) \psi\left(\mathbf{n}^{\prime}\right)} \mathbf{n} \cdot \mathbf{n}^{\prime}\right)+\lambda_{D}^{2}\right]^{2}} d \Omega^{\prime}, \\ G_{i}^{v, \operatorname{im}}(x, \mathbf{n}) & :=\int_{S^{2}} \frac{\mathscr{G}\left(\mathbf{k}, \mathbf{k}^{\prime}\right) \psi^{\frac{3}{2}\left(\mathbf{n}^{\prime}\right)}}{\left[\frac{2 m_{e} \mathscr{E}}{\hbar^{2} \gamma(\mathscr{E})}\left(\psi(\mathbf{n})+\psi\left(\mathbf{n}^{\prime}\right)-2 \sqrt{\psi(\mathbf{n}) \psi\left(\mathbf{n}^{\prime}\right)} \mathbf{n} \cdot \mathbf{n}^{\prime}\right)+\lambda_{D}^{2}\right]^{2}} \eta_{i}(\mathbf{n}) n_{i}^{\prime} d \Omega^{\prime}, \quad i=1,2,3 .\end{aligned}$

Obviously, these functions affect the angular integration of $\frac{\mathscr{G}\left(\mathbf{k}, \mathbf{k}^{\prime}\right)}{\left[\left|\mathbf{k}-\mathbf{k}^{\prime}\right|^{2}+\lambda_{D}^{2}\right]^{2}}$ and $\frac{\mathscr{G}\left(\mathbf{k}, \mathbf{k}^{\prime}\right)}{\left[\left|\mathbf{k}-\mathbf{k}^{\prime}\right|^{2}+\lambda_{D}^{2}\right]^{2}} v_{i}(\mathbf{k})$. Comparing with the polar optical scattering, here an additional simplification is shown due to the elasticity of this scattering, so $\mathscr{E}^{\prime}=\mathscr{E}$. Therefore, the production terms relative to velocities and energy fluxes have the same structure as that for polar optical scattering, with the elements of the matrix $\mathbf{Q}^{\text {(im) }}$ given by

$$
q_{i j}^{(\mathrm{im})}=\frac{\mathscr{K}^{(\mathrm{im})}}{2 \sqrt{2 m_{e}} \hbar J_{0} d_{0}} \int_{0}^{\infty} \frac{\mathscr{E}^{(i+j-2)} g^{2}(x)}{\gamma(x)} H_{5}(\mathscr{E}) e^{-\lambda_{W} \mathscr{E}} d \mathscr{E},
$$

with $\mathrm{H}_{5}$ given in Appendix A. 


\subsection{Production terms: non-polar optical phonon scattering}

In this section we consider inter-valley scattering due to non-polar phonons (3.6). After long and involved calculations we find, for the density and the energy production terms,

$$
\begin{aligned}
& \left(\begin{array}{l}
C_{n_{A}}^{(\mathrm{np})} \\
C_{W_{A}}^{(\mathrm{np})}
\end{array}\right)=\sum_{B \neq A} \frac{\sqrt{2} Z_{A B}\left(m_{e}\right)^{\frac{3}{2}} J_{0}^{A} J_{0}^{B}}{\hbar^{3}} N^{(\mathrm{np})} \mathscr{K}^{(\mathrm{np})}\left\{\frac { n _ { B } } { J _ { 0 } ^ { B } d _ { 0 } ^ { B } } \left[e^{-\lambda_{W}^{B} \Delta_{A B}^{+}+\frac{\varepsilon^{(\mathrm{np})}}{k_{B} T_{L}}}\right.\right. \\
& \quad \times \int_{0}^{\infty}\left(\begin{array}{l}
1 \\
\mathscr{E}+a_{A B}^{-}
\end{array}\right) e^{-\lambda_{W}^{B}\left(\mathscr{E}+a_{A B}^{-}\right)} H_{6, A B}\left(\mathscr{E}, a_{A B}^{-}, \Delta_{A B}^{+}\right) d \mathscr{E} \\
& \left.+e^{-\lambda_{W}^{B} \Delta_{A B}^{-}} \int_{0}^{\infty}\left(\begin{array}{l}
1 \\
\mathscr{E}+a_{A B}^{+}
\end{array}\right) e^{-\lambda_{W}^{B}\left(\mathscr{E}+a_{A B}^{+}\right)} H_{6, A B}\left(\mathscr{E}, a_{A B}^{+}, \Delta_{A B}^{-}\right) d \mathscr{E}\right] \\
& -\frac{n_{A}}{J_{0}^{A} d_{0}^{A}}\left[\int_{0}^{\infty}\left(\begin{array}{l}
1 \\
\mathscr{E}+a_{A B}^{-}
\end{array}\right) e^{-\lambda_{W}^{A}\left(\mathscr{E}+a_{A B}^{-}\right)} H_{6, A B}\left(\mathscr{E}, a_{A B}^{-}, \Delta_{A B}^{+}\right) d \mathscr{E}\right. \\
& \left.\left.+e^{\frac{\varepsilon^{(\mathrm{np})}}{k_{B} T_{L}}} \int_{0}^{\infty}\left(\begin{array}{l}
1 \\
\mathscr{E}+a_{A B}^{+}
\end{array}\right) e^{-\lambda_{W}^{A}\left(\mathscr{E}+a_{A B}^{+}\right)} H_{6, A B}\left(\mathscr{E}, a_{A B}^{+}, \Delta_{A B}^{-}\right) d \mathscr{E}\right]\right\}
\end{aligned}
$$

where $d_{0}^{A}=d_{0}\left(\lambda_{W}^{A}\right), \varepsilon^{(\mathrm{np})}=\hbar \omega^{(\mathrm{np})}, \Delta_{A B}^{ \pm}=\Delta_{A B} \pm \varepsilon^{(\mathrm{np})}, a_{A B}^{ \pm}=\max \left(0,-\Delta_{A B} \pm \varepsilon^{(\mathrm{np})}\right)$, and the function $H_{6, A B}$ can be found in Appendix A. The two positive terms in the previous expression depend through $\lambda_{W}^{B}$ on the macroscopic energy in valley $B$ and are gain terms, corresponding to the absorption and emission of a phonon respectively, while the negative terms depending on the macroscopic energy in valley $A$ are, of course, loss terms.

As regards the vector production terms, they have the same isotropic structure as those of the acoustic scattering, with the elements of the matrices $\mathbf{Q}_{A B}^{(\mathrm{np})}$ given by

$$
\begin{aligned}
q_{i j, A B}^{(\mathrm{np})}= & \frac{J_{0}^{B} Z_{A B}}{2 \hbar \sqrt{2 m_{e}} J_{0}^{A} d_{0}^{A}} N^{(\mathrm{np})} \mathscr{K}^{(\mathrm{np})} \int_{0}^{\infty}\left[\left(\mathscr{E}+a_{A B}^{-}\right)^{i+j-2} e^{-\lambda_{W}^{A}\left(\mathscr{E}+a_{A B}^{-}\right)}\right. \\
& \times H_{7, B}\left(\mathscr{E}, a_{A B}^{-}, \Delta_{A B}^{+}\right)+\left(\mathscr{E}+a_{A B}^{+}\right)^{i+j-2} e^{-\lambda_{W}^{A}\left(\mathscr{E}+a_{A B}^{+}\right)+\frac{\varepsilon^{(\mathrm{n})}}{k_{B} T_{L}}} \\
& \left.\times H_{7, B}\left(\mathscr{E}, a_{A B}^{+}, \Delta_{A B}^{-}\right)\right] d \mathscr{E}
\end{aligned}
$$

where the function $H_{7, B}$ is given in Appendix A.

We conclude this section by noting a property useful to cut the numerical computation time of the polar optical phonon and impurity production terms by a factor of eight.

Property 4 If $v\left(\mathbf{n}, \mathbf{n}^{\prime}\right)$ is an integrable function on $S^{2} \times S^{2}$, such that

- $v\left(-\mathbf{n},-\mathbf{n}^{\prime}\right)=v\left(\mathbf{n}, \mathbf{n}^{\prime}\right)$,

- $v\left(-n_{x},-n_{y}, n_{z}, n_{x}^{\prime}, n_{y}^{\prime}, n_{z}^{\prime}\right)=v\left(n_{x}, n_{y}, n_{z}, n_{x}^{\prime}, n_{y}^{\prime}, n_{z}^{\prime}\right)$,

- $v\left(-n_{x}, n_{y}, n_{z}, n_{x}^{\prime}, n_{y}^{\prime}, n_{z}^{\prime}\right)=v\left(n_{x}, n_{y}, n_{z}, n_{x}^{\prime}, n_{y}^{\prime}, n_{z}^{\prime}\right)$,

then

$$
\int_{S^{2} \times S^{2}} v\left(\mathbf{n}, \mathbf{n}^{\prime}\right) d \Omega d \Omega^{\prime}=8 \int_{Q^{1} \times S^{2}} v\left(\mathbf{n}, \mathbf{n}^{\prime}\right) d \Omega d \Omega^{\prime},
$$

where $Q^{1}$ is the portion of the unit sphere surface which lies in the first octant of $\mathbb{R}^{3}$. 
Table 2. Parameters of the band structure of $4 \mathrm{H}-\mathrm{SiC}$ and $6 \mathrm{H}-\mathrm{SiC}$

\begin{tabular}{lcccc}
\hline \hline Material & \multicolumn{2}{c}{ 4H-SiC } & \multicolumn{2}{c}{$6 \mathrm{H}-\mathrm{SiC}$} \\
\hline Conduction band valley & $\mathrm{M}, 1$ st c.b. & $\mathrm{M}, 2$ nd c.b. & L, 1st c.b. & L, 2nd c.b. \\
Effective mass $\left(m_{e}\right)$ & $0.29,0.58,0.33$ & $0.90,0.58,0.33$ & $0.22,0.90,1.43$ & $0.22,0.90,1.43$ \\
Valley energy minimum $(\mathrm{eV})$ & 0 & 0.14 & 0 & 0 \\
$\alpha\left(\mathrm{eV}^{-1}\right)$ & 0.117 & 0.058 & 0.039 & 0.039 \\
Number of equivalent & 3 & 3 & 3 & 3 \\
$\quad$ valleys & & & & \\
\hline \hline
\end{tabular}

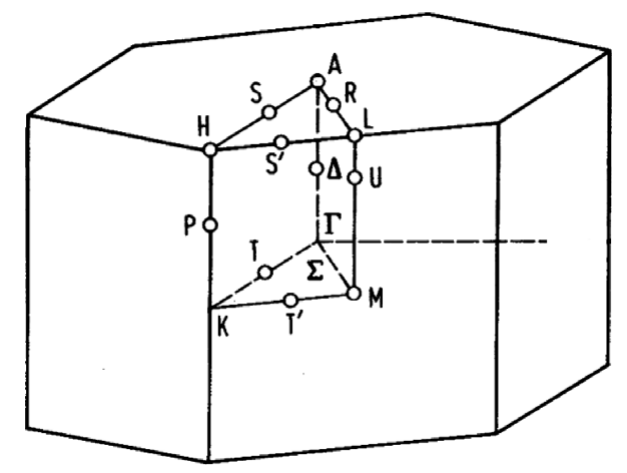

FIGURE 1. Brillouin zone of hexagonal lattice.

The integrands in the production terms of the polar optical phonon and impurity scatterings satisfy the hypotheses under which Property 4 holds.

\section{Numerical simulations of bulk $4 \mathrm{H}-\mathrm{SiC}$ and $6 \mathrm{H}-\mathrm{SiC}$}

In [1] the model with isotropic dispersion relations (in the following indicated as IB model) has been applied to the cases of bulk $\mathrm{GaN}$ and $4 \mathrm{H}-\mathrm{SiC}$. Here we show that the model with anisotropic dispersion relations (in the following indicated as AB model) is more appropriate to describe the behaviour of anisotropic semiconductors such as the hexagonal polytypes of silicon carbide whose lattice is shown in Figure 1. This is proved by the fact that the behaviour of the drift velocity and the average energy with respect to the electric field strongly depends on the direction of the latter. Silicon carbide is an important semiconductor material because of its high saturation velocity, large thermal conductivity, high breakdown voltages and high Schottky barriers. These properties make it very useful in high temperature, high frequency and high power devices. As far as we are aware, there have been few attempts to construct hydrodynamical models, without any free adjustable parameter or ad hoc closure, which describe the electric behaviour of such a material. In particular, it appears that to date no attempt has been made to take into account strong anisotropy. 
Table 3. Bulk material parameters

\begin{tabular}{lcccccccc}
\hline \hline & $\rho$ & $\epsilon_{s}$ & $\epsilon_{\infty}$ & $v_{s}$ & $\Xi_{d}$ & $\hbar \omega^{(p)}$ & $\hbar \omega^{(\mathrm{np})}$ & $D_{t} K$ \\
\hline $4 \mathrm{H}-\mathrm{SiC}$ & 3.2 & 9.7 & 6.5 & 13,730 & 15 & 120 & 85.4 & $7 \times 10^{8}$ \\
6H-SiC & 3.2 & 9.66 & 6.5 & $\begin{array}{c}13,730 \\
\mathrm{~m} / \mathrm{s}\end{array}$ & $\begin{array}{c}17.5 \\
\mathrm{eV}\end{array}$ & $\begin{array}{c}120 \\
\mathrm{meV}\end{array}$ & $\begin{array}{c}85.4 \\
\mathrm{meV}\end{array}$ & $\begin{array}{c}6 \times 10^{8} \\
\mathrm{meV}\end{array}$ \\
\hline \hline
\end{tabular}

\subsection{Bulk 4H-SiC}

As regards the band structure of $4 \mathrm{H}-\mathrm{SiC}$, different results can be found in the literature. Here we have used those given in Pennington \& Goldsman [21], that is, we have considered the valleys around the minima at the symmetry point $M$ of the two lowest conduction bands. Because of crystal symmetries there are three equivalent $M$ points, therefore the total number of valleys taken into account is six, and their parameters are listed in Table 2. In particular, in this table, the masses along the principal directions $\mathrm{M}-\mathrm{K}, \mathrm{M}-\Gamma$ and $\mathrm{M}-\mathrm{L}$ (in the following respectively indicated by $x, y$ and $z$ ) are given, $m_{e}$ is the free electron mass, and $\epsilon_{0}$ is electrical permittivity in a vacuum. If in the bulk case homogeneous initial conditions are taken with zero initial velocities and energy fluxes, the moment system reduces to a system of ordinary differential equations (ODEs) with time as the only independent variable. In this case, the Poisson equation is solved taking the total electron population equal to the doping concentration, and the electrostatic potential with a linear profile between the two boundary values, while the moment system reduces to

$$
\begin{aligned}
& \frac{d n_{A}}{d t}=C_{n_{A}}\left(W_{1}, W_{2}, \ldots\right)+\sum_{B \neq A} n_{B} C_{n_{A}}\left(W_{B}\right), \\
& \frac{d}{d t}\left(n_{A} V_{A}^{i}\right)+q n_{A} \sum_{j} E_{j} G_{A}^{(0) i j}=n_{A} c_{11}^{A, i}\left(W_{A}\right) V_{A}^{i}+n_{A} c_{12}^{A, i}\left(W_{A}\right) S_{A}^{i}, \\
& \frac{d}{d t}\left(n_{A} W_{A}\right)+q n_{A} \sum_{j} E_{j} V_{A}^{j}=C_{W_{A}}\left(W_{1}, W_{2}, \ldots\right)+\sum_{B \neq A} n_{B} C_{W_{A}}\left(W_{B}\right), \\
& \frac{d}{d t}\left(n_{A} S_{A}\right)^{i}+q n_{A} \sum_{j} E_{j} G_{A}^{(1) i j}=n_{A} c_{21}^{A, i}\left(W_{A}\right) V_{A}^{i}+n_{A} c_{22}^{A, i}\left(W_{A}\right) S_{A}^{i},
\end{aligned}
$$

where the index A runs over the valleys; moreover, for each valley the vector equations are written using the respective basis. All the scattering mechanisms reported in Section 3 are taken into account and the material parameters can be found in Table 3, following the data in [15].

All the terms relative to the density and the energy production have been summed into the first addenda in equations (7.1) and (7.3), except the gain terms of inter-valley scattering, which depend on the energy of other valleys, different from $A$, involved in the scattering and are represented by the sums on the right-hand side of the equations. The production terms are numerically computed for a discrete number of values in a suitable range of macroscopic energies and the values of interest during the numerical 
(a)

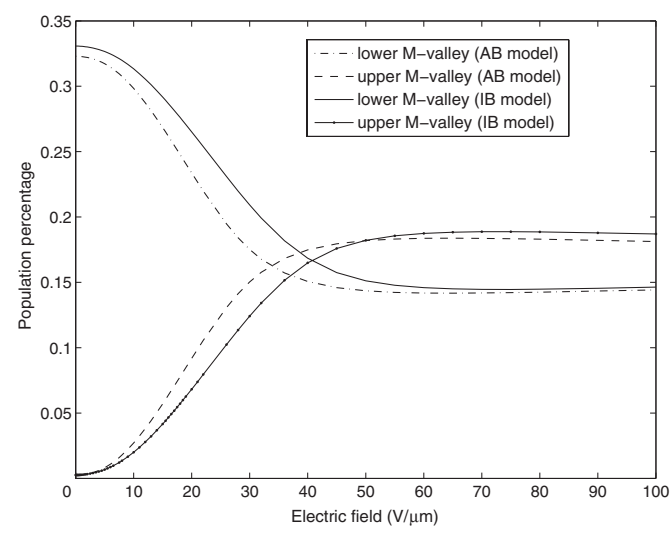

(b)

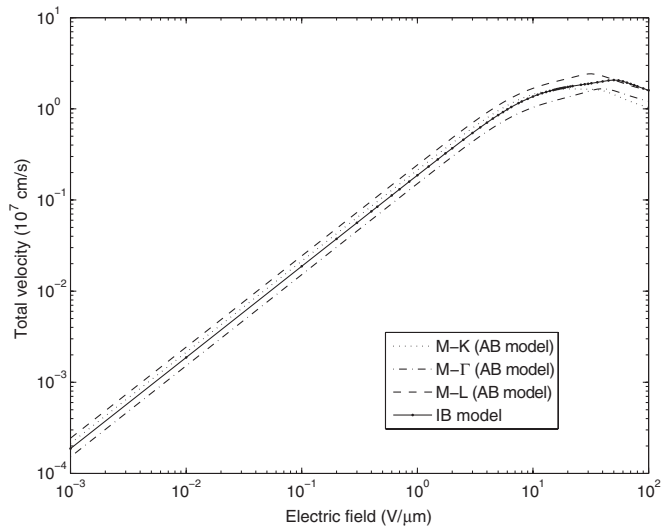

FIGURE 2. 4H-SiC. (a) Valley occupancies $\left(\frac{n_{A}}{\sum_{A} n_{A}}\right)$ versus the applied electric field directed along $\mathrm{M}-\mathrm{K}$. (b) Total velocity versus the applied electric field in the principal directions. The doping density is $\mathrm{N}_{D}=10^{19} / \mathrm{cm}^{3}$.

computation of the solution to the system (7.1)-(7.4) are evaluated by a stepwise linear interpolation. As said, the computation of the production terms relative to the scattering both with the polar optical phonons and impurities is very expensive, in fact it takes around 20 hours on a Workstation with a 8-GB RAM and a $2.67-\mathrm{GHz}$ Intel(R) Xeon(R) X3450 CPU. However, these computations are done once for all and can be used for all kinds of devices. In particular, for the angular integration a suitable numerical method has been exploited, which has been tested verifying that if the masses are equal, then the results of the isotropic model are recovered. The ODE system is numerically solved by a 4th order Runge-Kutta method for 60 values of applied electric field going in modulus from $10^{-3}$ to $100 \mathrm{~V} / \mu \mathrm{m}$. Three directions of the field are considered, that is the principal directions of one of the three equivalent M-valleys. The steady state is reached in less than $5 \mathrm{ps}$ and the program requires a total run-time of less than $30 \mathrm{~s}$ on a laptop with an i7-2630QM processor.

The results are shown in Figures 2-3. All the main characteristics of $4 \mathrm{H}-\mathrm{SiC}$ are qualitatively and quantitatively well described. At low fields, up to $4-5 \mathrm{~V} / \mu \mathrm{m}$, the behaviour of the total average velocity, which is defined as

$$
\mathbf{V}=\frac{1}{n} \sum_{A} n_{A} \mathbf{V}_{A}
$$

is linear with high slope, and the velocity reaches its maxima, which are about $2.04 \times 10^{7}$, $1.94 \times 10^{7}$ and $2.42 \times 10^{7} \mathrm{~cm} / \mathrm{s}$ respectively in the $x, y$ and $z$ directions, at about the fields 40,40 and $32 \mathrm{~V} / \mu \mathrm{m}$. This latter fact together with Figure $2(\mathrm{~b})$ is enough to show that the differences according to the direction of the electric field are relevant, which makes it clear that taking into account the anisotropy of the energy dispersion relations is very important. Also, the behaviour of the valley occupancies, as shown in Figure 2(a), is different from that foreseen by the IB model, in fact the population inversion occurs at a lower electric field than in the isotropic case. There is a remarkable discrepancy, Figure 3(a), 
(a)

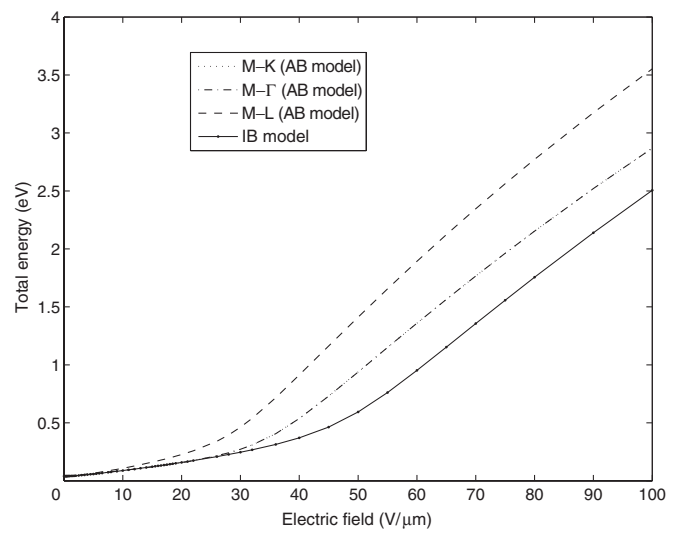

(b)

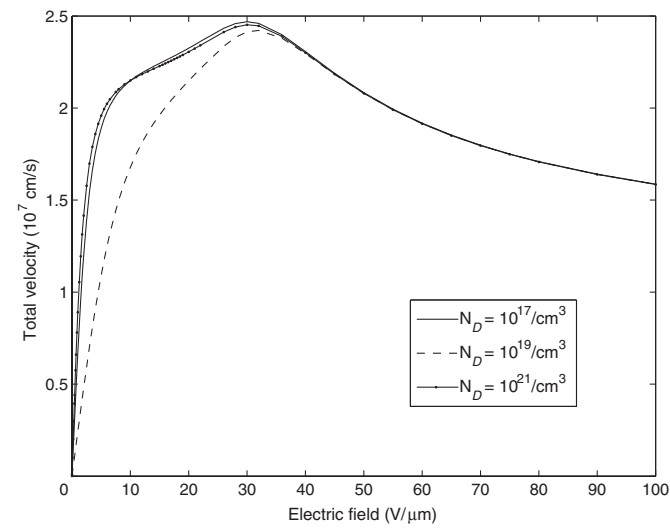

FIGURE 3. 4H-SiC. (a) Total average energy (measured from the bottom of the 1 st $M$-valley) versus the applied electric field in the principal directions, for $\mathrm{N}_{D}=10^{19} / \mathrm{cm}^{3}$. The results relative to the $\mathrm{M}-\mathrm{K}$ and $\mathrm{M}-\Gamma$ directions are practically indistinguishable. (b) Total velocity versus the applied electric field directed along $\mathrm{M}-\mathrm{L}$ for three values of $\mathrm{N}_{D}$.
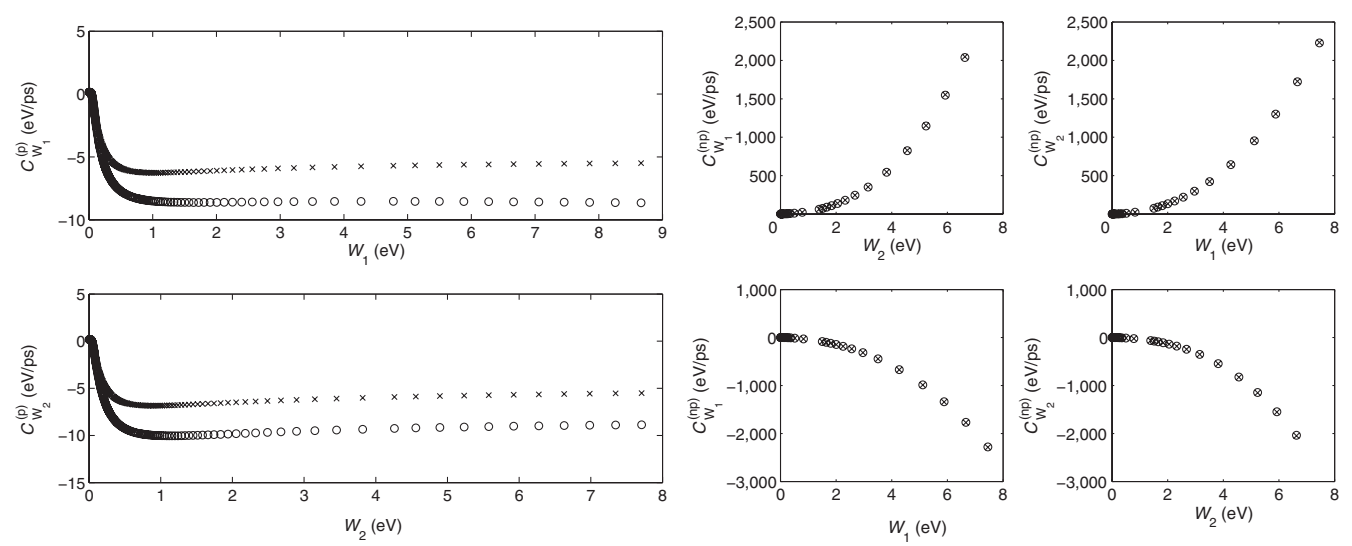

FIGURE 4. 4H-SiC. In the two figures on the left: polar optical energy productions (for the two $\mathrm{M}$-valleys). In the four figures on the right: gain (upper figures) and loss (lower figures) terms of the non-polar optical energy productions (for the two M-valleys) (6.12). The figures compare the results for the two models, circles referring to the IB model and crosses to the AB model.

between the predictions of the two models in the behaviour of the total mean energy, which is given by

$$
W=\frac{1}{n} \sum_{A} n_{A}\left(W_{A}+\mathscr{E}_{A}^{(0)}-\mathscr{E}_{1}^{(0)}\right) .
$$

This might also be due to the different overlap factor used in this paper as can be deduced from the fact that relevant differences in the energy production terms are present only for the polar optical scattering, see Figure 4. Figure 5 shows the non-polar optical density productions. As regards the production terms for the velocities and energy fluxes, also for them the main differences are in the polar optical and impurity scattering, which are 

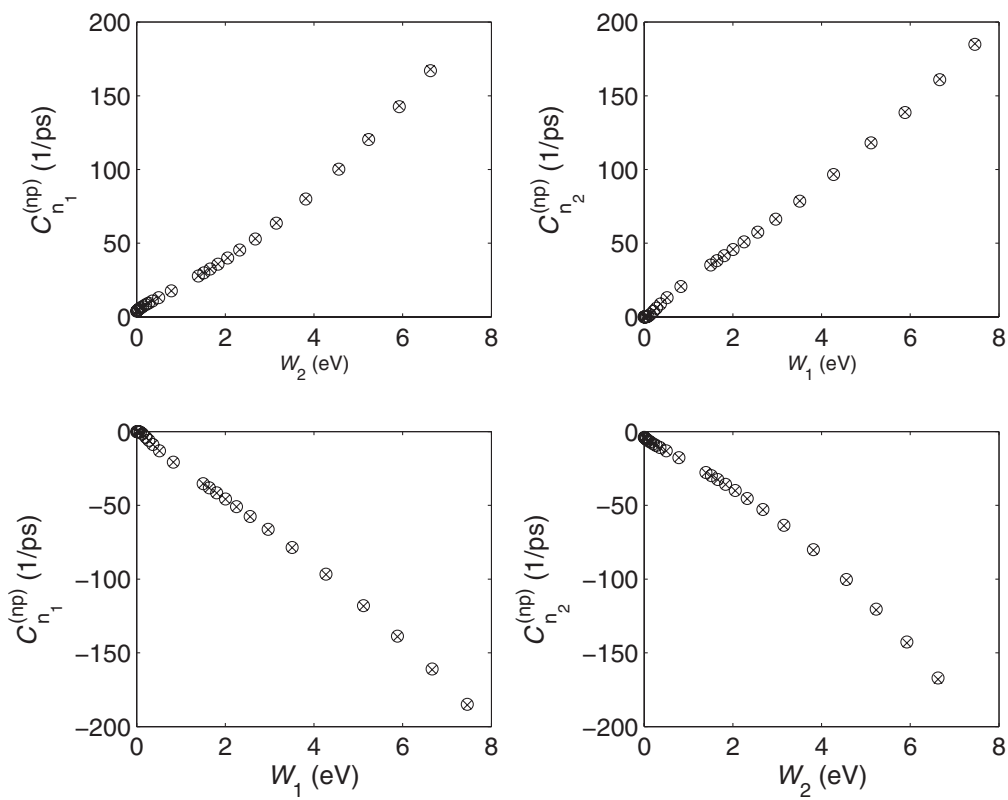

FIGURE 5. 4H-SiC. Non-polar optical density productions (for two M-valleys).Circles refer to the IB model, and crosses to the AB model.
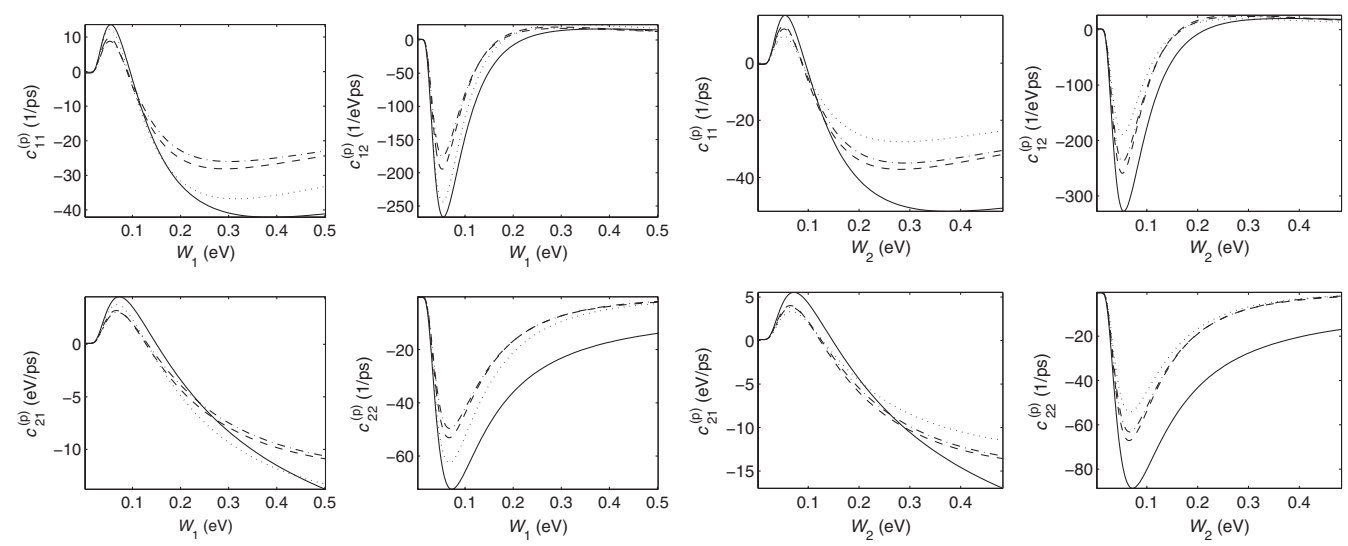

Figure 6. $4 \mathrm{H}$-SiC, elements of the matrices $\mathbf{C}_{A}^{(p), i}, i=1,2,3$, for the polar optical scattering. In the four figures on the left: lower valley. In the four figures on the right: higher valley. Symbols have the same meaning as in Figure 2(b), referring to the main directions and to the type of model.

anisotropic and for which the overlap factor (6.7) is used, while for the acoustic and non-polar optical scattering the differences are irrelevant, see Figures 6-9.

At last in Figure 3(b), we show how the behaviour of the drift velocity varies at different doping concentrations, and the model manages to capture non-monotonicity with increasing impurity concentration typical of $\mathrm{SiC}$. 

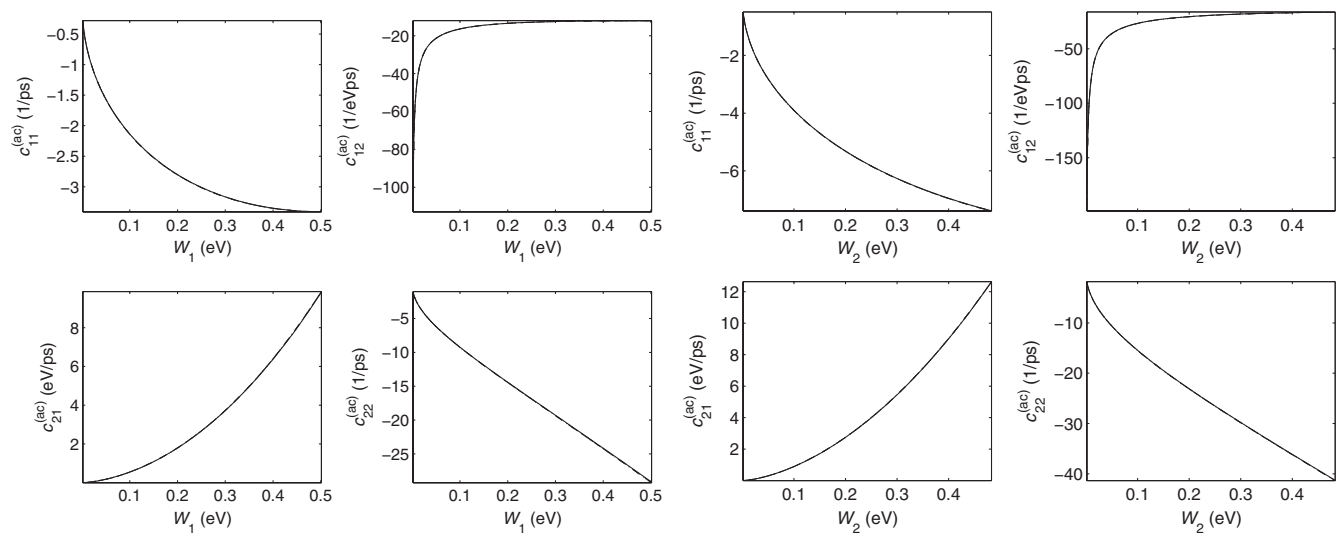

FIGURE 7. 4H-SiC, acoustic scattering. In the four figures on the left: velocity and energy flux production terms (lower valley). In the four figures on the right: velocity and energy flux production terms (higher valley). For this scattering, differences in different directions and between the two models are practically indiscernible.
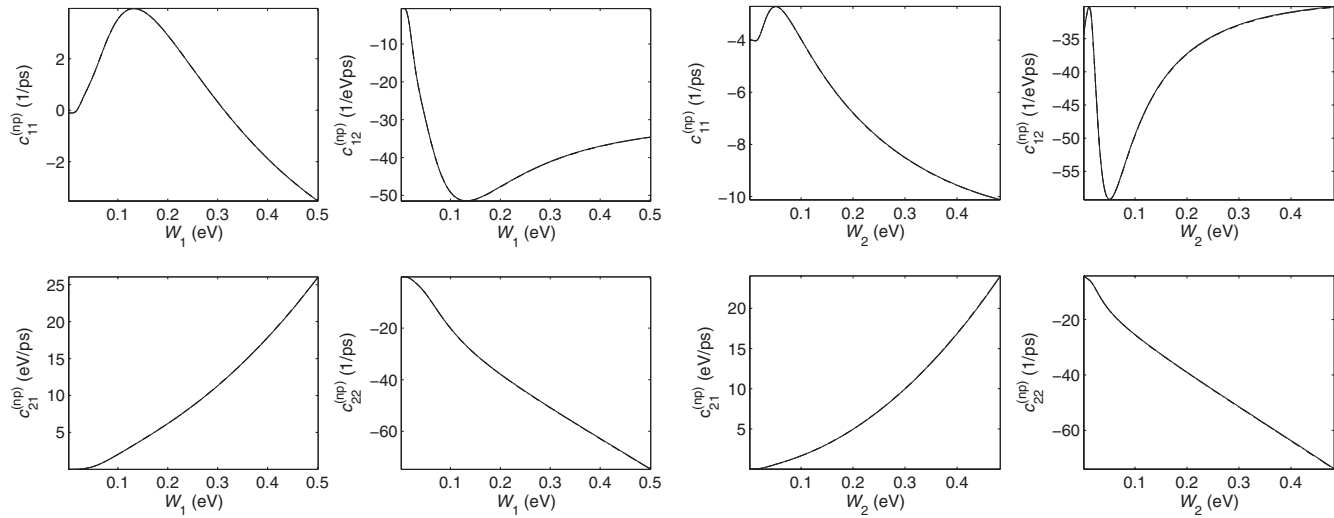

FIGURE 8. 4H-SiC, non-polar optical scattering. In the four figures on the left: velocity and energy flux production terms (lower valley). In the four figures on the right: velocity and energy flux production terms (higher valley). Also, in this case differences in different directions and between the two models are practically indiscernible.

\subsection{H-Bulk SiC}

Also in this case for the band structure we have used the results of [21]. In particular, we have considered the minima of the two lowest conduction bands to occur at the $L$ symmetry point and used a number of equivalent valleys equal to three. Therefore, also in this case, the total number of valleys is six, and furthermore the two lowest conduction bands degenerate around L. The parameters are given in Table 2, where in particular the masses along the principal directions $\mathrm{L}-\mathrm{H}, \mathrm{L}-\mathrm{A}$ and $\mathrm{L}-\mathrm{M}$ can be found. The results are shown in Figures 10-11 and are qualitatively analogous to those for $4 \mathrm{H}-\mathrm{SiC}$, therefore the same comments remain valid also in this case. For a better comparison with the experimental values found by von Muench and Pettenpaul [24], in Figure 10(a) we have highlighted by squares the velocities corresponding to the values of the electric field 

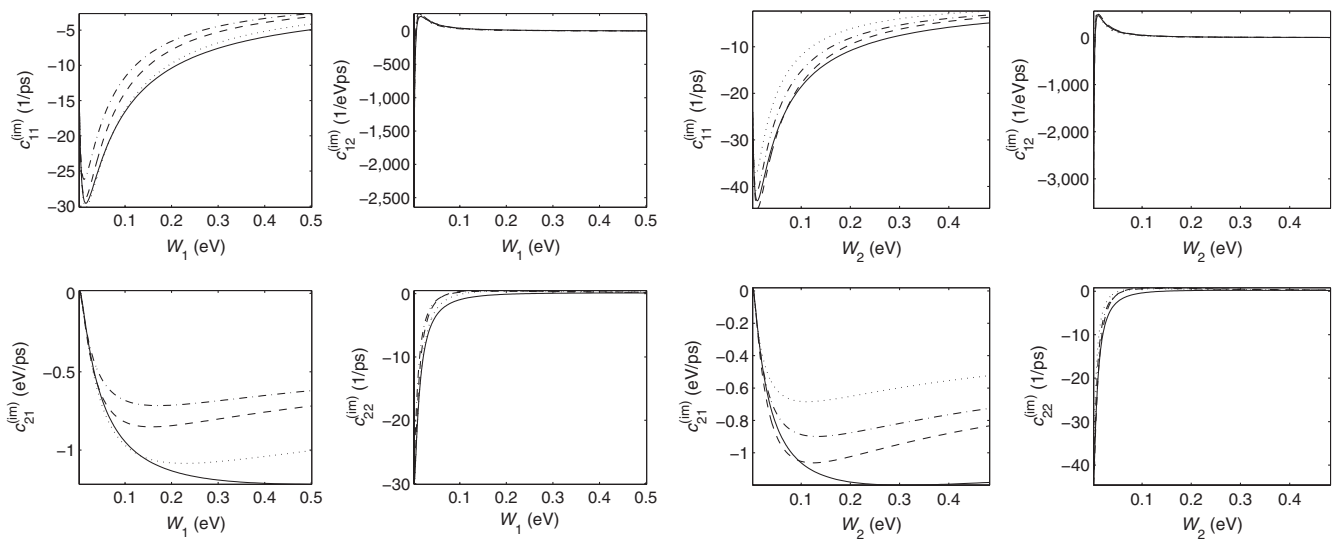

FIGURE 9. 4H-SiC, impurity scattering. In the four figures on the left: velocity and energy flux production terms (lower valley). In the four figures on the right: velocity and energy flux production terms (higher valley). Symbols have the same meaning as in Figure 2(b). $\mathrm{N}_{D}=10^{19} / \mathrm{cm}^{3}$.

(a)

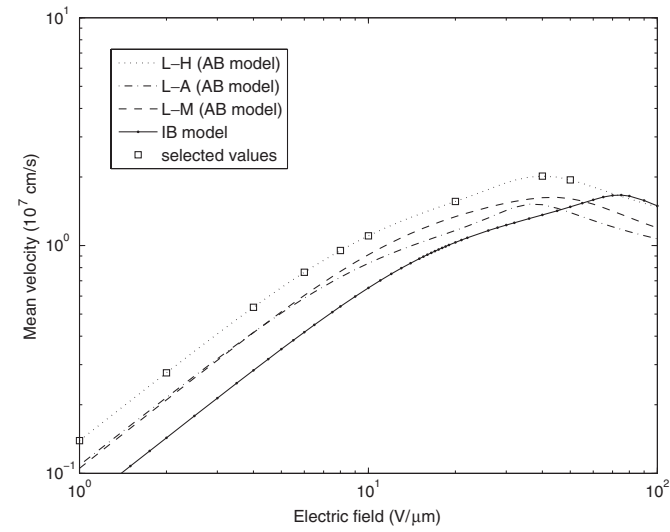

(b)

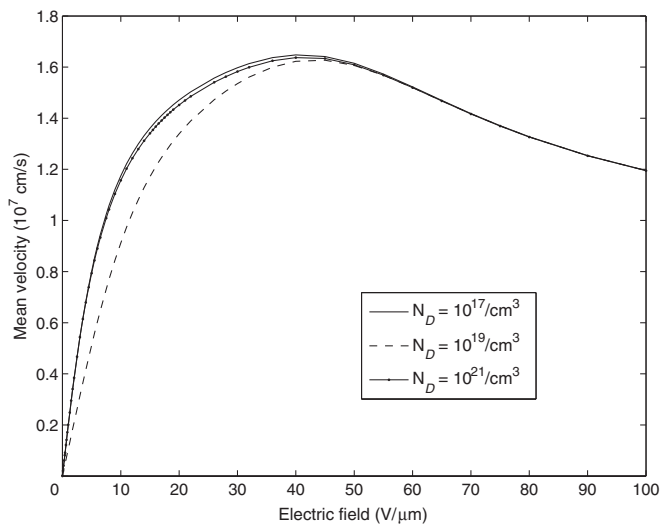

Figure 10. 6H-SiC. (a) Total velocity versus the applied electric field in the principal directions, $\mathrm{N}_{D}=10^{19} / \mathrm{cm}^{3}$. (b) Total velocity versus the applied electric field directed along L-M for three values of $\mathrm{N}_{D}$.

reported in [15]. Eventually, Figure 12 shows that, as known, the anisotropy of $4 \mathrm{H}-\mathrm{SiC}$ is lower than that of $6 \mathrm{H}-\mathrm{SiC}$.

\section{Conclusions}

In this paper we present a hydrodynamical model for charge transport in semiconductors which, to the best of our knowledge, is the first to take into account the dependence of charge effective mass on space direction. This is very important for highly anisotropic semiconductors such as $4 \mathrm{H}-$ and $6 \mathrm{H}-\mathrm{SiC}$. The numerical results for these latter semiconductors, shown in the paper, are in good qualitative and quantitative agreement with the Monte Carlo results in the literature. In our opinion the results could be further improved, 


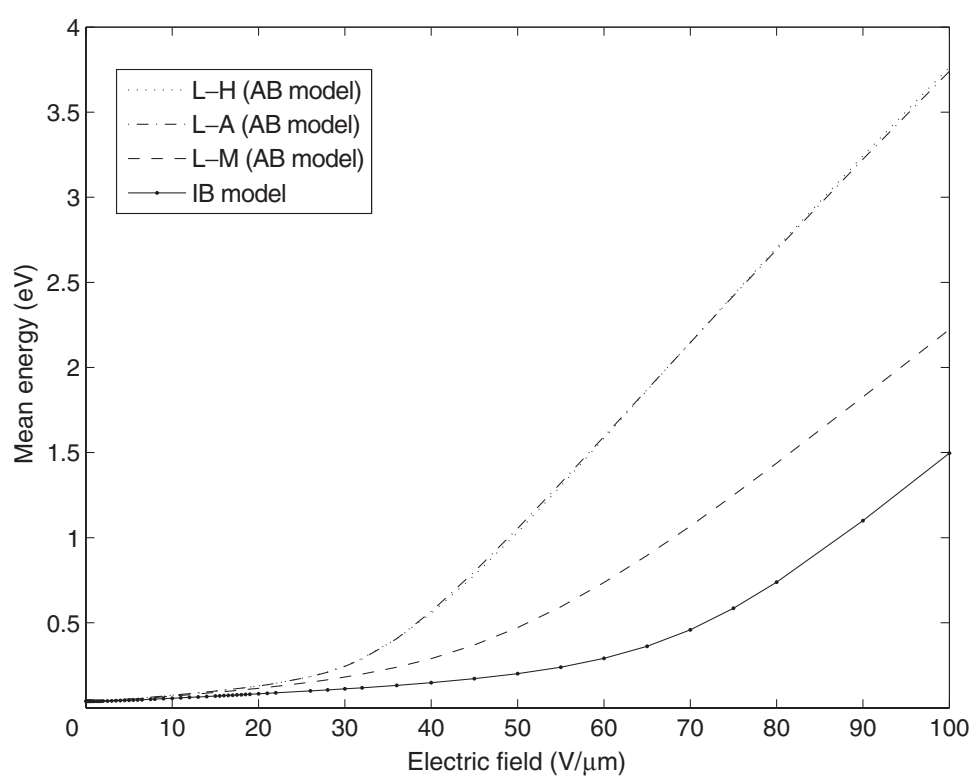

FIGURE 11. 6H-SiC. Total average energy (measured from the bottom of the first c.b.) versus the applied electric field along the three principal axes. The results relative to the $\mathrm{L}-\mathrm{H}$ and $\mathrm{L}-\mathrm{A}$ directions are practically indistinguishable.

(a)

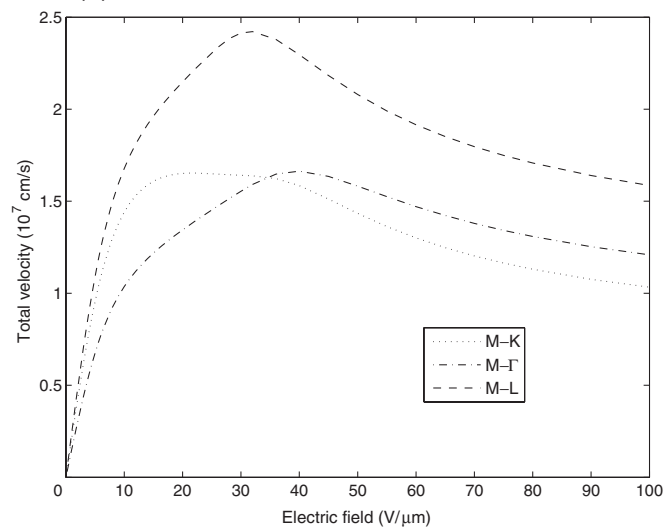

(b)

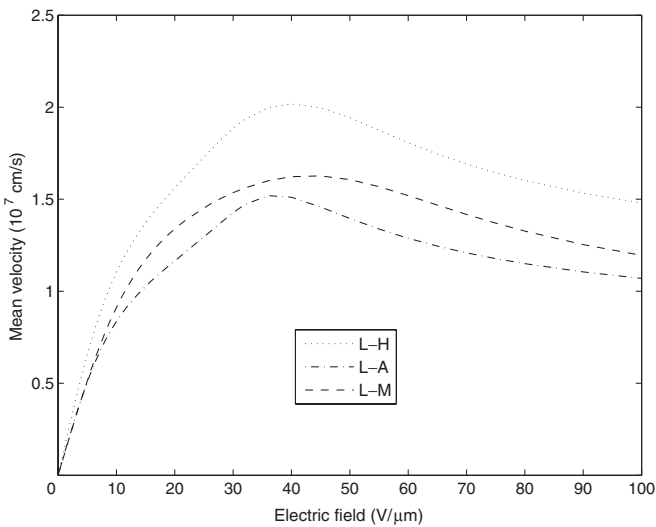

FIGURE 12. (a) $4 \mathrm{H}-\mathrm{SiC}$, total velocity versus the applied electric field directed along the principal directions. (b) $6 \mathrm{H}-\mathrm{SiC}$, total velocity versus the applied electric field directed along the principal directions. $\mathrm{N}_{D}=10^{19} / \mathrm{cm}^{3}$.

since they strongly depend on the parameters of the band structure and the scattering rates for which several different values can be found in the literature.

\section{Acknowledgements}

Giuseppe Alì and Giovanni Mascali acknowledge the financial support from P.R.A., University of Calabria. Vittorio Romano acknowledges the financial support from P.R.A., 
University of Catania and the P.R.I.N. project 2010 'Kinetic and macroscopic models for particle transport in gases and semiconductors: analytical and computational aspects', and by the 'MIUR PON' AMBITION POWER project. Giuseppe Ali, Giovanni Mascali and Vittorio Romano also acknowledge the financial support from EU ENIAC LAST POWER project.

\section{Appendix A}

In this section we list the functions that appear in various production terms

$$
\begin{aligned}
H_{1}(x) & :=\sqrt{x} \frac{\gamma(x)-x \dot{\gamma}(x)}{\gamma(x)^{\frac{5}{2}}}, \\
H_{2, i}(x, y) & :=\frac{g^{2}(x) H_{1}(y)}{\gamma(x) H_{1}(x)} \int_{S^{2}} G^{s, p}(x, y, \mathbf{n}) \eta_{i}^{2}(\mathbf{n}) n_{i}^{2} \psi^{\frac{3}{2}}(\mathbf{n}) d \Omega, \\
H_{3, i}(x, y) & :=e^{\hbar \omega^{(p)}\left(\frac{1}{k_{B} T_{L}}-\lambda_{W}\right)} \frac{g^{2}(y) H_{1}(x)}{\gamma(y) H_{1}(y)} \int_{S^{2}} G^{s, p}(y, x, \mathbf{n}) \eta_{i}^{2}(\mathbf{n}) n_{i}^{2} \psi^{\frac{3}{2}}(\mathbf{n}) d \Omega, \\
H_{4, i}(x, y) & :=\frac{g(x) g(y)}{\sqrt{\gamma(x) \gamma(y)}} \sqrt{x y} \int_{S^{2}} G_{i}^{v, p}(x, y, \mathbf{n}) \eta_{i}(\mathbf{n}) n_{i} \psi^{\frac{3}{2}}(\mathbf{n}) d \Omega, \\
H_{5}(x) & :=\int_{S^{2}}\left[G^{s, i m}(x, \mathbf{n}) \eta_{i}^{2}(\mathbf{n}) n_{i}^{2} \psi^{\frac{3}{2}}(\mathbf{n})-G_{i}^{v, i m}(x, \mathbf{n}) \eta_{i}(\mathbf{n}) n_{i} \psi^{\frac{3}{2}}(\mathbf{n})\right] d \Omega, \\
H_{6, A B}(x, y, z) & :=H_{1, A}(x+y) H_{1, B}(x+y+z), \\
H_{7, B}(x, y, z) & :=\frac{8 m_{e}(x+y)^{2}}{\hbar^{2}} H_{1, B}(x+y+z)
\end{aligned}
$$

\section{References}

[1] Alì, G., Mascali, G., Romano, V. \& Torcasio, R. C. (2012) A hydrodynamic model for covalent semiconductors with applications to GaN and SiC. Acta Appl. Mat. 103(1), 335348, doi:10.1007/s10440-012-9747-6.

[2] Anile, A. M. \& Romano, V. (1999) Non-parabolic band transport in semiconductors: Closure of the moment equations. Contin. Mech. Thermodyn. 11, 307

[3] Dreyer, W. (1987) Maximisation of the entropy in non-equilibrium. J. Phys. A: Math. Gen. 20, 6505 .

[4] Fawcett, W., Boardman, A. D. \& Swain, S. (1970) Monte Carlo determination of electron transport properties in gallium arsenide. J. Phys. Chem. Solids 31(9), 1963.

[5] Huelm, M., Nilsson, H.-E., Martinez, A., Brennan, K. F. \& Bellotti, E. (2003) Monte Carlo study of high-field carrier transport in $4 \mathrm{H}-\mathrm{SiC}$, including band-to-band tunneling. J. Appl. Phys. 93(2), 1099.

[6] Jacoboni, C. \& Lugli, P. (1989) The Monte Carlo Method for Semiconductor Device Simulation, Springer-Verlag, Wien, New York, NY.

[7] Jaynes, E. T. (1957) Information theory and statistical mechanics. Phys. Rev. 106, 620.

[8] La Rosa, S., Mascali, G. \& Romano, V. (2009) Exact maximum entropy closure of the hydrodynamical model for Si semiconductors: The 8-moment case. SIAM J. Appl. Math. 70, 710 .

[9] Majorana, A. (1993) Equilibrium solutions of the non-linear Boltzmann equation for an electron gas in a semiconductor. IL Nuovo Cimento B 108(8), 871-877. 
[10] Mascali, G. (2002) Maximum entropy principle in relativistic radiation hydrodynamics II: Compton and double Compton scattering. Contin. Mech. Thermodyn. 14(6), 549.

[11] Mascali, G. \& Romano, V. (2005) Si and GaAs mobility derived from a hydrodynamical model for semiconductors based on the maximum entropy principle. Physica A 352(2-4), 459.

[12] Mascali, G. \& Romano, V. (2010) Hydrodynamic subband model for semiconductors based on the maximum entropy principle. IL Nuovo Cimento C 33(1), 155-163.

[13] Mascali, G. \& Romano, V. (2011) A hydrodynamical model for holes in silicon semiconductors: The case of non-parabolic warped bands. Math. Comp. Modelling 53(1-2), 213.

[14] Mascali, G., Romano, V. \& Sellier, J. M. (2005) MEP parabolic hydrodynamical model for holes in silicon semiconductors. IL Nuovo Cimento B 120(2), 197.

[15] Mickevicius, R. \& ZhaO, J. H. (1998) Monte Carlo study of electron transport in SiC. J. Appl. Phys. 83(6), 3161.

[16] Muscato, O. (2001) The Onsager reciprocity principle as a check of consistency for semiconductor carrier transport models. Physica A 289(3-4), 422.

[17] Muscato, O. \& Di Stefano, V. (2008) Modeling heat generation in a sub-micrometric $n^{+}-n-n^{+}$ silicon diode. J. Appl. Phys. 104(12), 124501.

[18] Muscato, O. \& Di Stefano, V. (2011) Local equilibrium and off-equilibrium thermoelectric effects in silicon semiconductors. J. Appl. Phys. 110(9), 093706.

[19] Muscato, O., Pidatella, R. M. \& Fischetti, M. V. (1998) Monte Carlo and hydrodynamic simulation of a one dimensional $\mathrm{n}^{+}-\mathrm{n}-\mathrm{n}^{+}$silicon diode. VLSI Design 6 (1-4), 247.

[20] Nilsson, H. E., Huelm, M., Fröjdh, C., Persson, C., Sannemo, U. \& Petersson, C. S. (1999) Full band Monte Carlo simulation of electron transport in 6H-SiC. J. Appl. Phys. 86, 965.

[21] Pennington, G. \& Goldsman N. (2004) Consistent calculations for n-type hexagonal SiC inversion layers. J. Appl. Phys. 95(8), 4223-4234.

[22] Romano, V. (2000) Non-parabolic band transport in semiconductors: Closure of the production terms in the moment equations. Cont. Mech. Thermodyn. 12, 31.

[23] Tomizawa, K. (1993) Numerical Simulation of Submicron Semiconductor Devices. Artech House, Boston, MA.

[24] von Muench, W. \& Pettenpaul, J. (1977) Saturated electron drift velocity in $6 \mathrm{H}$ silicon carbide. J. Appl. Phys. 48, 4823.

[25] Zhao, J. H., Gruzinskis, V., Luo, Y., Weiner, M., Pan, M., Shiktorov, P. \& Starikov, E. (2000) Monte Carlo simulation of 4H-SiC IMPATT diodes. Semicond. Sci. Technol. 15, 1093. 